\title{
HIGH DYNAMIC RANGE VLBI OBSERVATIONS OF THE 3C120 RADIO JET
}

\author{
J.M. Benson ${ }^{1}$, R.C. Walker ${ }^{1}$, T.W.B. Muxlow ${ }^{2}$ \\ P.N. Wilkinson ${ }^{2}$, R. Booth ${ }^{3}$, G. Pilbratt ${ }^{3}$, S.C. Unwin ${ }^{4}$ \\ ${ }^{1}$ NRAO, Charlottesville, VA \\ ${ }^{2}$ NRAL, Jodrell Bank, Macclesfield, UK \\ ${ }^{3}$ Onsala Space Obs., Onsala, Sweden \\ ${ }^{4}$ OVRO, Caltech, Pasadena, CA
}

3C 120 is a nearby radio galaxy ( $z=0.033)$ that shows superluminal motions in its radio jet within one parsec of the nucleus. The apparent velocities of the moving features are approximately 1.5 mas/yr. This object is of particular interest because it is clearly identified with an N-type galaxy, unlike all other superluminal radio sources which are only seen in quasars and BL Lac objects. Because of the close proximity of $3 C 120$, we have been able to make a series of VLBI and VLA images at stepped resolutions, and thereby map the radio jet continuously from the radio nucleus $\left(0.5 \mathrm{~h}^{-1}\right.$ parsec) into the diffuse $400 \mathrm{~h}^{-1} \mathrm{kpc}$ structure.

We observed 3C 120 at two epochs with VLBI arrays consisting of 14 and 18 stations. The latter was the so-called 'World Radio Array' experiment. The observations were conducted at $18 \mathrm{~cm}$. At the time of the second VLBI run, $18 \mathrm{~cm}$ observations were also obtained with the MERLIN array. The large number of stations and carefully organized calibration observations enabled us to make radio images having dynamic ranges of nearly 1000:1 (peak flux to off-source rms). The off-source noise level in the Apr $1984 \mathrm{map}$ is $0.5 \mathrm{mJy} / \mathrm{beam}$, roughly twice the theoretically derived noise level.

There are four basic results from the maps shown in the figure. 1). We see, in both VLBI maps, a well collimated jet running eastward from the radio core for about $75 \mathrm{~h}^{-1} \mathrm{pc}$. Beyond that distance, the jet suddenly expands into an opening angle of 30 degrees. The superluminal components, whose motions we monitor at $6 \mathrm{~cm}$, are blended together in the region within 20 mas of the core. 2). The 1982 and 1984 images both distinctly show emission knots and twists in the jet before it begins to expand. The individual features are clearly seen in both epochs and are essentially spatially coincident. 3). The emission knot lying between 40 and 60 mas from the core may have moved outward at approximately $1.2 \mathrm{mas} / \mathrm{yr}$, nearly the same velocity as the superluminal features near the radio core. The jet may therefore exhibit superluminal velocities up to the point where it begins to expand. 4). The VLBI and MERLIN images taken together provide a link 31 
in spatial resolution between the superluminal region $\left(10 \mathrm{~h}^{-1} \mathrm{pc}\right)$ and the kiloparsec features which have been completely imaged by the VLA. The radio jet is continuously connected from the radio core to the most distant $400 \mathrm{~h}^{-1} \mathrm{kpc}$ emission region.
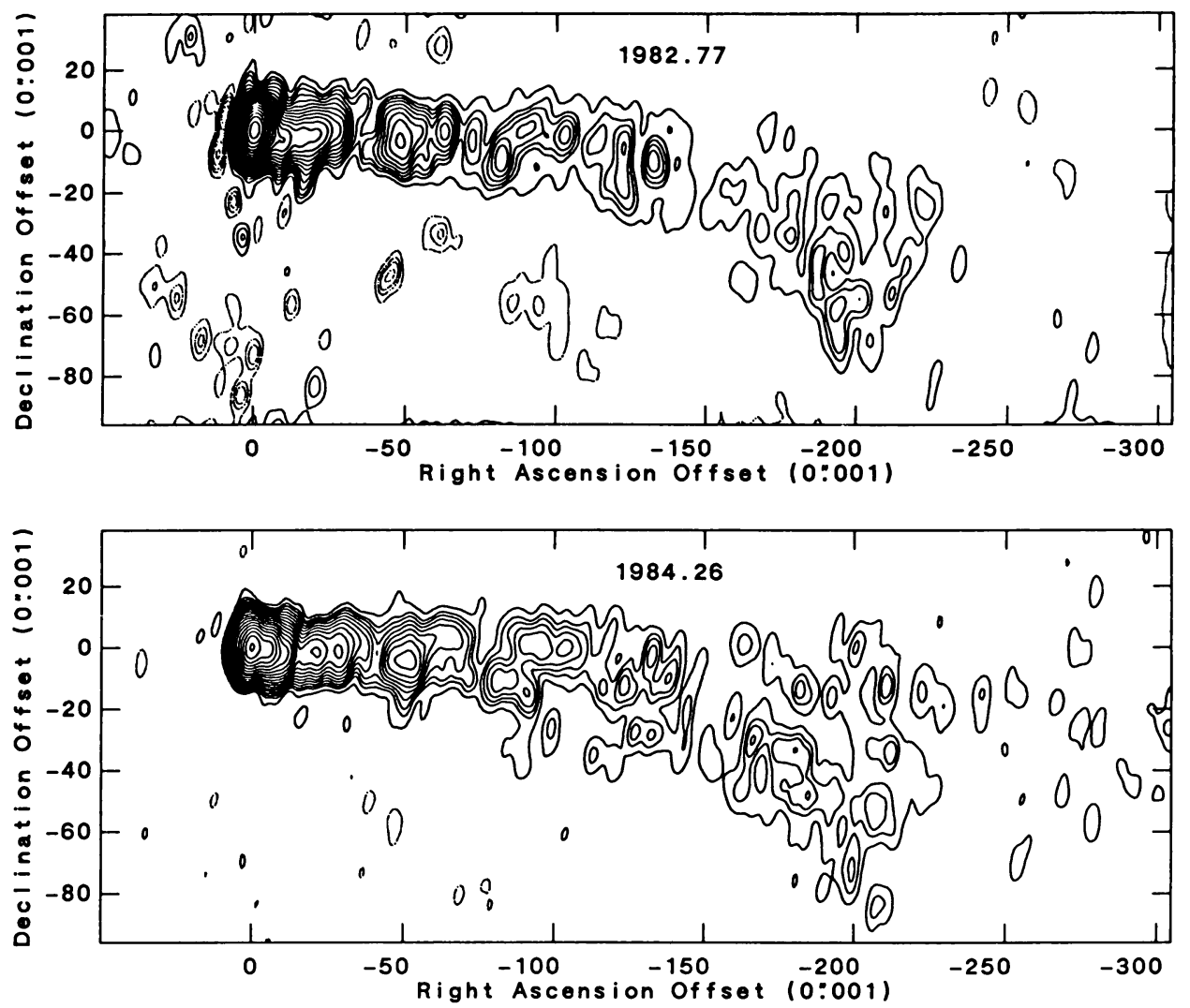

Fig. 1. Full resolution maps of $3 \mathrm{C} 120$ at $18 \mathrm{~cm}$ in October 1982 and April 1984. The convolving beam is 3 by 6 milliarcsec in position angle $-10 \mathrm{deg}$. The contour levels are logarithmic : $-6.2,-4.5,-$ $3.2,-2.3,-1.2,1.2,2.3,3.2,4.5,6.2,8.6,12.0,16.7,23.2,32.2$, $44.8,62.2,86.4,120.0,166.9,231.6,321.6,447.6,621.6,864.0$, 1666.8 milliJanksies. 